\title{
Quantification of light-enhanced ionic transport in lead iodide perovskite thin films and its solar cell applications
}

\author{
Yi-Cheng Zhao ${ }^{1}$, Wen-Ke Zhou ${ }^{1}$, Xu Zhou ${ }^{1}$, Kai-Hui Liu ${ }^{1,2}$, Da-Peng Yu ${ }^{1,2}$ and Qing Zhao ${ }^{1,2}$
}

Ionic transport in organometal halide perovskites is of vital importance because it dominates anomalous phenomena in perovskite solar cells, from hysteresis to switchable photovoltaic effects. However, excited state ionic transport under illumination has remained elusive, although it is essential for understanding the unusual light-induced effects (light-induced self-poling, photo-induced halide segregation and slow photoconductivity response) in organometal halide perovskites for optoelectronic applications. Here, we quantitatively demonstrate light-enhanced ionic transport in $\mathrm{CH}_{3} \mathrm{NH}_{3} \mathrm{Pbl}_{3}$ over a wide temperature range of $17-295 \mathrm{~K}$, which reveals a reduction in ionic transport activation energy by approximately a factor of five (from 0.82 to $0.15 \mathrm{eV}$ ) under illumination. The pure ionic conductance is obtained by separating it from the electronic contribution in cryogenic galvanostatic and voltage-current measurements. On the basis of these findings, we design a novel light-assisted method of catalyzing ionic interdiffusion between $\mathrm{CH}_{3} \mathrm{NH}_{3} \mathrm{I}$ and $\mathrm{Pbl}_{2}$ stacking layers in sequential deposition perovskite synthesis. X-ray diffraction patterns indicate a significant reduction of $\mathrm{Pbl}_{2}$ residue in the optimized $\mathrm{CH}_{3} \mathrm{NH}_{3} \mathrm{Pbl}$ thin film produced via lightassisted sequential deposition, and the resulting solar cell efficiency is increased by over $100 \%(7.5 \%-15.7 \%)$ with little $\mathrm{PbI}_{2}$ residue. This new method enables fine control of the reaction depth in perovskite synthesis and, in turn, supports light-enhanced ionic transport.

Light: Science \& Applications (2017) 6, e16243; doi:10.1038/lsa.2016.243; published online 5 May 2017

Keywords: activation energy; ion migration; light; perovskite

\section{INTRODUCTION}

Electrical conduction in materials can be classified into two categories, electronic and ionic, depending on the conducting species. A mixed conductor is a material that possesses both electronic and ionic conductivity, and the recently emerging family of organometal halide perovskites, $\mathrm{ABX}_{3}\left(\mathrm{~A}: \mathrm{CH}_{3} \mathrm{NH}_{3}(\mathrm{MA}) / \mathrm{NH}_{2} \mathrm{CH}=\mathrm{NH}(\mathrm{FA}) / \mathrm{Cs}, \mathrm{B}: \mathrm{Pb} / \mathrm{Sn}\right.$, $\mathrm{X}: \mathrm{I} / \mathrm{Cl} / \mathrm{Br}$ ), has proven to be mixed conductors ${ }^{1-12}$. Ion migration has been reported to be one of the main mechanisms responsible for anomalous I-V hysteresis and poor stability in perovskite solar cells. For optoelectronic applications of these organometal halide perovskites, light-induced effects will inevitably occur in optoelectronic devices such as solar cells, light-emitting diodes (LEDs) and lasers ${ }^{13-15}$. Under working conditions and continuous light illumination, the long-term output of perovskite-based solar cells is reported to be unstable. LED applications also exhibit a decrease in efficiency after some period of operation. It is reasonable to speculate that these perovskites possess different properties in their excited state under photoexcitation, which deserve further investigation to gain a better understanding of the optoelectronic properties of perovskites, especially with regard to these unusual light-induced effects ${ }^{10,16-23}$.
However, to date, excited state ion migration for perovskites under photoexcitation has rarely been reported in $\mathrm{CH}_{3} \mathrm{NH}_{3} \mathrm{PbI}_{3}\left(\mathrm{MAPbI}_{3}\right)$.

Herein, we demonstrate a systematic and quantitative study of lightdependent ionic transport in $\mathrm{MAPbI}_{3}$ film over a wide range of temperatures $(17-295 \mathrm{~K})$ and light intensities $\left(0-20 \mathrm{~mW} \mathrm{~cm}^{-2}\right)$, by means of combined cryogenic galvanostatic ${ }^{6,24}$ and voltage-current measurements. Distinct from the band-like nature of the highmobility electronic transport in this material, the ionic transport exhibits an obvious hopping mechanism with varying activation energies under different illumination intensities. The activation energy for ionic transport shows a significant decrease by approximately a factor of five (from 0.82 to $0.15 \mathrm{eV}$ ) as the light intensity increases from 0 to $20 \mathrm{~mW} \mathrm{~cm}^{-2}$. To the best of our knowledge, this result is the first demonstration of light-dependent ionic transport in organometal halide perovskites. This light-enhanced ion migration can explain the photo-induced giant dielectric constant in the lowfrequency regime and the photo-instability in perovskite-based devices as well as the light-induced halide segregation in mixed perovskites. We further demonstrate that this property can be utilized to manipulate the synthesis kinetics of perovskites through lightassisted ion migration. The results yield an obvious increase in solar

${ }^{1}$ State Key Laboratory for Mesoscopic Physics and Electron Microscopy Laboratory, School of Physics, Peking University, Beijing 100871, China and ${ }^{2}$ Collaborative Innovation Center of Quantum Matter, Beijing 100084, China

Correspondence: KH Liu, Email: khliu@pku.edu.cn; Q Zhao, Email: zhaoqing@pku.edu.cn

Received 6 July 2016; revised 24 September 2016; accepted 18 October 2016; accepted article preview online 21 October 2016 
cell efficiency due to reduced interfacial $\mathrm{PbI}_{2}$. Our findings provide important fundamental insights into the excited state properties of perovskite film, which deserves future investigation in inorganic halide perovskites, even for the whole ionic crystal family.

\section{MATERIALS AND METHODS}

$\mathrm{MAPbI}_{3}$ was prepared from an MAI/PbAc 2 (Sigma-Aldrich, Shanghai, China) (3:1) precursor solution (215 and $172 \mathrm{mg} \mathrm{ml}^{-1}$ for MAI and $\mathrm{PbAc}_{2}$, respectively) with an additional $4 \mu \mathrm{l}$ of $\mathrm{H}_{3} \mathrm{PO}_{2}$ (Aladdin, Shanghai, China). Then, the solution was spin coated onto a plasmacleaned $\mathrm{FTO} / \mathrm{TiO}_{2}$ substrate. The film was sintered at $100{ }^{\circ} \mathrm{C}$ for $50 \mathrm{~min}$ in ambient air ( $\sim 30 \%$ relative humidity). The hole transport material (HTM) was then spin coated onto the perovskite film at $3000 \mathrm{rpm}$ for $40 \mathrm{~s}$. The spin-coating formulation was prepared by dissolving $72.3 \mathrm{mg}$ of 2,2',7,7'-tetrakis(N,N-p-dimethoxy-phenylamino)-9,9'-spirobifluorene (spiro-MeOTAD) purchased from Yingkou OPV Tech New Energy Co., Ltd. (Shanghai, China), $30 \mu \mathrm{l}$ of 4-tert-butylpyridine (tBP) and $20 \mu \mathrm{l}$ of a stock solution of $520 \mathrm{mg} \mathrm{ml}^{-1}$ lithium bis(trifluoromethylsulphonyl)imide (Li-TFSI) in acetonitrile in $1 \mathrm{ml}$ of chlorobenzene. Finally, 90-nm-thick gold electrodes were deposited via thermal evaporation. The active electrode area was fixed at $9 \mathrm{~mm}^{2}$.

For the light-assisted method of sequential deposition used to prepare the perovskite, $\mathrm{PbI}_{2}$ in $\mathrm{DMF}$ at a concentration of $450 \mathrm{mg} \mathrm{ml}^{-1}$ was spin coated onto an $\mathrm{FTO} / \mathrm{TiO}_{2}$ substrate $\left(3000 \mathrm{rpm}\right.$ for $30 \mathrm{~s}$ ), followed by drying at $90^{\circ} \mathrm{C}$ for $10 \mathrm{~min}$. An $\mathrm{MAI} / \mathrm{MACl}\left(45 / 5 \mathrm{mg} \mathrm{ml}^{-1}\right)$ mixed solution was then spin coated onto the prepared $\mathrm{PbI}_{2}$ film (4000 rpm for $30 \mathrm{~s}$ ), followed by annealing at $110^{\circ} \mathrm{C}$ for $10 \mathrm{~min}$. Three batches of samples were prepared for comparison: annealing without light ( $0 \mathrm{~min})$, annealing with $5 \mathrm{~min}$ of light exposure $(5 \mathrm{~min})$, and annealing with $10 \mathrm{~min}$ of light exposure (10 min). The light intensity was $\sim 40 \mathrm{~mW} \mathrm{~cm}^{-2}$. All samples were treated with the same annealing temperature and duration $\left(110^{\circ} \mathrm{C} /\right.$ $10 \mathrm{~min}$ ) and were then solvent annealed with DMF at $100^{\circ} \mathrm{C}$ for $40 \mathrm{~min}$. For XRD characterizations, these as-prepared samples were directly mounted on the sample stage. For the preparation of solar cell devices, these samples were covered with an electron-blocking layer of spiro-MeOTAD and a gold layer, as described above.

$\mathrm{X}$-ray powder diagrams were recorded using an X'PertMPD PRO from PANalytical equipped with a ceramic tube $(\mathrm{Cu}$ anode, $\lambda=1.54060 \mathrm{~A}$ ), a secondary graphite (002) monochromator and an RTMS X'Celerator detector, operating in the Bragg-Brentano geometry. For a description of the steady-state photoluminescence (PL) spectra and time-resolved fluorescence spectra, which were recorded using a high-resolution streak camera system (Hamamatsu C10910), and the current-voltage measurements, please refer to reference 12. X-ray photoelectron spectra were measured using a PHI Quantera SXM system (Chigasaki, Kanagawa, Japan) under a vacuum of $1.0 \times 10^{-7}$ Torr, after using an $\mathrm{Ar}^{+}$gun at $2 \mathrm{kV} / 30 \mathrm{~s}$ to clean the sample surface.

For high-field electric poling experiments, four pairs of electrodes were prepared on a glass substrate using a hard silica template with four pairs of square apertures (with $50-\mu \mathrm{m}$ gaps and $500-\mu \mathrm{m}$ widths). The thickness of the gold electrodes was $\sim 0.8 \mu \mathrm{m}$. After $30 \mathrm{~s}$ of oxygen plasma treatment to remove residual organic material to enhance the wetting properties of the plates, an $\mathrm{MAPbI}_{3}$ precursor solution (MAI/ $\mathrm{PbAc}_{2}$ (Sigma-Aldrich) (3:1)) was spin coated onto the substrate, followed by annealing at $100{ }^{\circ} \mathrm{C}$ for $50 \mathrm{~min}$ in ambient air $(30 \%$ relative humidity). In the high-field poling experiments, optical images under different illuminations were recorded in ambient air at room temperature $(\sim 300 \mathrm{~K})$. In situ observations of the ion drift were performed by placing the samples under an optical microscope
(Olympus BX51) coupled to a colored CMOS digital camera, model GCI-070103 (Daheng New Epoch Technology, Inc., Shanghai, China). The optical microscope was operating in the reflection mode with the sample illuminated from the bottom.

The current-voltage (I-V) and galvanostatic characteristics of the samples were obtained using an Agilent B2900 Series precision source/ measure unit (Beijing, China). The time duration for each galvanostatic measurement was $0.1 \mathrm{~s}$, and the applied currents were $0.02,0.04$, $0.2,0.8$ and $2 \mathrm{nA}$ for $0,0.05,1,5$ and $20 \mathrm{~mW} \mathrm{~cm}^{-2}$, respectively, considering that the gradually increasing conductivity under stronger illumination would have resulted in very weak signals if the same current of $0.02 \mathrm{nA}$ had been used. After these data were collected, we used the procedures described in the main text to extract the ionic and electronic conductances. Finally, the ideal formula below was used to obtain the conductivity: $\sigma=\frac{G L}{s} \times 10^{9} \approx 0.52 \times 10^{9} \mathrm{G}\left(\mu \mathrm{S} \mathrm{cm}^{-1}\right)$, where $G$ is the conductance, $s$ is the cross-sectional area, and $L$ is the gap in the lateral device architecture.

For cryogenic electrical experiments, we used a small silica template to prepare Au electrodes confined to the sample stage in the chamber, which left a gap on the perovskite film of $50 \mu \mathrm{m}$ in width. The cryogenic experiments were conducted in a cryostation (Montana model $\mathrm{C} 2$ ) at temperatures ranging from $17 \mathrm{~K}$ to room temperature. The lateral device was directly mounted on the He-cooled cryostat with a temperature controller, in a high-vacuum box at $0.9 \mu$ Torr. The device was measured at increments of temperature from 17 to $295 \mathrm{~K}$, with stabilization for more than $10 \mathrm{~min}$ at each temperature. The temperature increase was found to result in an increase of less than $2{ }^{\circ} \mathrm{C}$ in the temperature of the device under $20 \mathrm{~mW} \mathrm{~cm}^{-2}$ illumination (230V MI-150 Fiber Optic Illuminator).

\section{RESULTS AND DISCUSSION}

High-field poling behavior of the $\mathrm{Au} / \mathrm{MAPbI}_{3} / \mathrm{Au}$ lateral structure First, for macroscopic in situ detection of ionic motion under different illumination conditions, we performed high-field electrical poling experiments using an $\mathrm{Au} / \mathrm{MAPbI}_{3} / \mathrm{Au}$ lateral device structure with a $50-\mu \mathrm{m}$ gap filled with $\mathrm{MAPbI}_{3}$. Because a change in the contrast of optical images recorded under an optical microscope can be observed as a result of mobile ions under high-field poling ${ }^{1,2,25}$, a $100-\mathrm{V}$ bias was applied to this device under three different light intensities $(0,5$ and $20 \mathrm{~mW} \mathrm{~cm}^{-2}$ ). The dynamic process was recorded on video using a time accelerated mode (Supplementary Movie 1-3), nine snapshots of which are presented in Figure 1. Under dark conditions (Figure 1a), no contrast change of the perovskite film induced by ion migration was observed, whereas under illumination, a black line formed after $10 \mathrm{~s}$ of poling (Figure 1b). Moreover, many plane-dendrite structures formed under stronger illumination (Figure 1c), implying more severe ionic motion. Similar evidence of light-enhanced ionic motion was also observed in vacuum under high-field poling (Supplementary Fig. S1). As illustrated in Figure $1 \mathrm{~d}, \mathrm{I}^{-}$reduction and a subsequent $\mathrm{I}_{2}$ volatilization process occurred at the cathode under high-field poling, and $\mathrm{MA}^{+}$could also move toward the anode, where it evaporated away in the form of $\mathrm{CH}_{3} \mathrm{NH}_{2}$, assisted by moisture and illumination. The gradual transformation ${ }^{25,26}$ from $\mathrm{MAPbI}_{3}$ into $\mathrm{PbI}_{2}$ (Supplementary Fig. S2) was accompanied by $\mathrm{MA}^{+}$migration from the cathode to the anode, which together created the observed threads in the perovskite film. This scenario was further confirmed by comparing the elemental distributions before and after high-field poling, which revealed no discernible ionic pile-up near the electrodes after high-field poling and the formation of many voids around these black threads (Supplementary Fig. S2). 


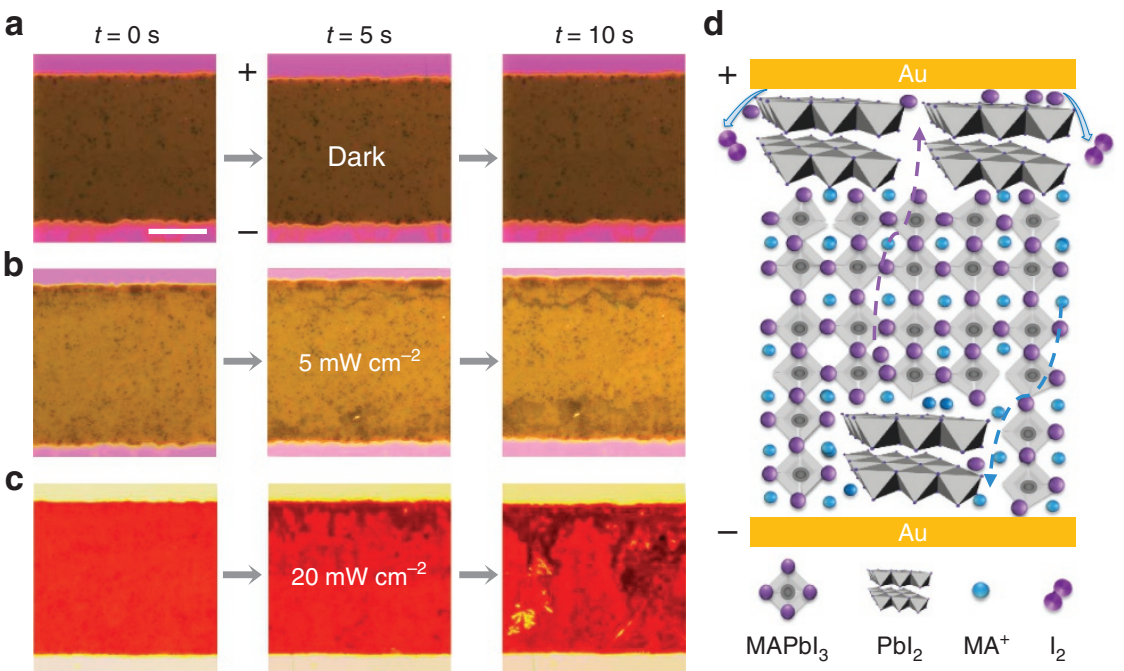

Figure 1 Optical dynamic images of perovskite film under electric poling and illumination of various intensities in ambient air at room temperature. (a) The

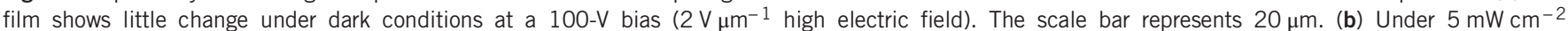
illumination, the film shows black threads at the cathode, with black dots expanding at the anode after $10 \mathrm{~s}$ of high-field poling. (c) Under $20 \mathrm{~mW} \mathrm{~cm}^{-2}$ illumination, the film shows black threads and plane-dendrite structures forming at the cathode and expanding from the cathode to the anode after $10 \mathrm{~s}$ of poling. (d) Schematic illustration of the migration of $\mathrm{I}^{-}$anions and $\mathrm{MA}^{+}$cations through the film. The temperature of the perovskite film under light illumination was monitored with a precision of $1{ }^{\circ} \mathrm{C}$ by an infrared thermometer.

\section{Characterization of light-enhanced ionic transport}

With these macroscopic findings in mind, we proceeded to a quantitative evaluation of the change in the energy barrier for ion migration under a varying light intensity. Gold was used for the electrodes to guarantee ohmic contact because of the p-type nature of our perovskite film (Supplementary Fig. S3). The time-resolved PL spectrum confirmed the high intrinsic quality of the perovskite film, with a $\sim 270$ ns lifetime (Supplementary Fig. S3). To extract the ionic conductivity $\left(\sigma_{\text {ion }}\right)$, a current-voltage $(\mathrm{I}-\mathrm{V})$ scan was first performed to obtain the mixed conductivity $\sigma_{\text {total }}$ in the perovskite film at a $50 \mathrm{~V} \mathrm{~s}^{-1}$ scan rate in the $\mathrm{Au} / \mathrm{MAPbI}_{3} / \mathrm{Au}$ device. Here, the ionic accumulation effect was essentially negligible because the scanning period was much shorter than the relaxation time for ionic motion (Figure 2a and 2c). Then, we performed galvanostatic characterization with a sufficiently weak current to separate the pure electronic conductivity $\sigma_{\mathrm{e}}$ from the mixed conductivity $\sigma_{\text {total }}$, which is a standard technique in mixed conductor investigations ${ }^{24,27,28}$. When the current was switched on from 0 to $20 \mathrm{pA}$, the measured resistance immediately reached an initial value and then gradually increased to a stable value (Figure $2 \mathrm{~b}$ ). The ionic migration and accumulation are the rate-determining processes, whereas the equilibrium value was determined only by the electronic conductivity $\sigma_{\mathrm{e}}$. As the schematic illustrations (Figure 2c-2e) show, initially, both electrons and ions contributed to the conductance, corresponding to the fast-scan I-V measurements and region I in Figure $2 \mathrm{~b}$. Subsequently, these mobile ions were gradually depleted because of ionic accumulation at the two sides of the film, resulting in fewer ions contributing to the conductance of the perovskite film (region II and Figure 2d). Finally, the conductance reached a stable value, with all mobile ions blocked at the boundary; then, only the electronic conductance remained (region III and Figure 2e). The double-layer capacitance effect was very weak $^{2,5}$ because of the small bias voltage applied in the measurements and the high resistance of the perovskite film. An 800-nm short-pass filter was used here to exclude the influence of phonons on the ionic motion under illumination. Finally, the ionic contribution was extracted from the mixed conductivity by subtracting the electronic conductance from the mixed conductance: $\sigma_{\text {ion }}=\sigma_{\text {total }}-\sigma_{\mathrm{e}}$.

Through the analysis described above, we extracted the pure electronic and ionic conductivities over a wide range of temperatures (17 to $295 \mathrm{~K}$ ) and light intensities (0 to $20 \mathrm{~mW} \mathrm{~cm}^{-2}$ ) using the apparatus shown in Figure 3a, in which incident light was shed on the sample through an objective lens (numerical aperture $(\mathrm{NA})=0.2$ ). Figure $3 \mathrm{~b}$ presents the results of our galvanostatic measurements at different temperatures, where the gradual increase in the resistance corresponds to the slow depletion of the ionic conductivity in the perovskite thin film. In addition, the relaxation times required to reach the steady-state resistance reflect the kinetic constant for ion migration at different temperatures from 100 to $295 \mathrm{~K}$, which is denoted by $k=1 / \tau$. The ionic relaxation time is inversely correlated with temperature because of the reduced vibration frequency at lower temperature.

The pure electronic resistance decreases exponentially with increasing temperature, and an obvious linear region corresponding to defect ionization appears above $150 \mathrm{~K}$ (Figure 3c). The corresponding ionization energies for these defects in the perovskite film are 92, 84, 73, 44 and $53 \mathrm{meV}$ under illumination intensities of $0,0.05,1,5$ and $20 \mathrm{~mW} \mathrm{~cm}^{-2}$, respectively. The results indicate shallow-level defects in the sample. The reduction in the ionization energy level under illumination is attributed to a screening effect induced by the photoexcited carriers. We also observe a conductivity flip below $70 \mathrm{~K}$ under illumination. This behavior strongly implies an inverse power dependence of the mobility on the temperature, $\mu \propto T^{-3 / 2}$, at temperatures ranging from 150 to $17 \mathrm{~K}^{10,29}$, originating from the acoustic phonon scattering in perovskites. The corresponding electronic conductivity is described by the semi-empirical formula below:

$$
\begin{aligned}
\sigma_{\mathrm{e}}= & n e \mu=N_{\mathrm{d}} \times \exp \left(-\beta E_{\mathrm{a}}\right) \times e\left(\mu_{0}+a T^{-3 / 2}\right) \\
& +f\left(I_{0}\right) \times e \times\left(\mu_{0}+a T^{-3 / 2}\right)
\end{aligned}
$$

where $N_{\mathrm{d}}$ is the total defect concentration, $\beta$ is $1 / k_{\mathrm{B}} T, k_{\mathrm{B}}$ is the Boltzmann constant, $E_{\mathrm{a}}$ is the activation energy for trap defects, $a$ is a 

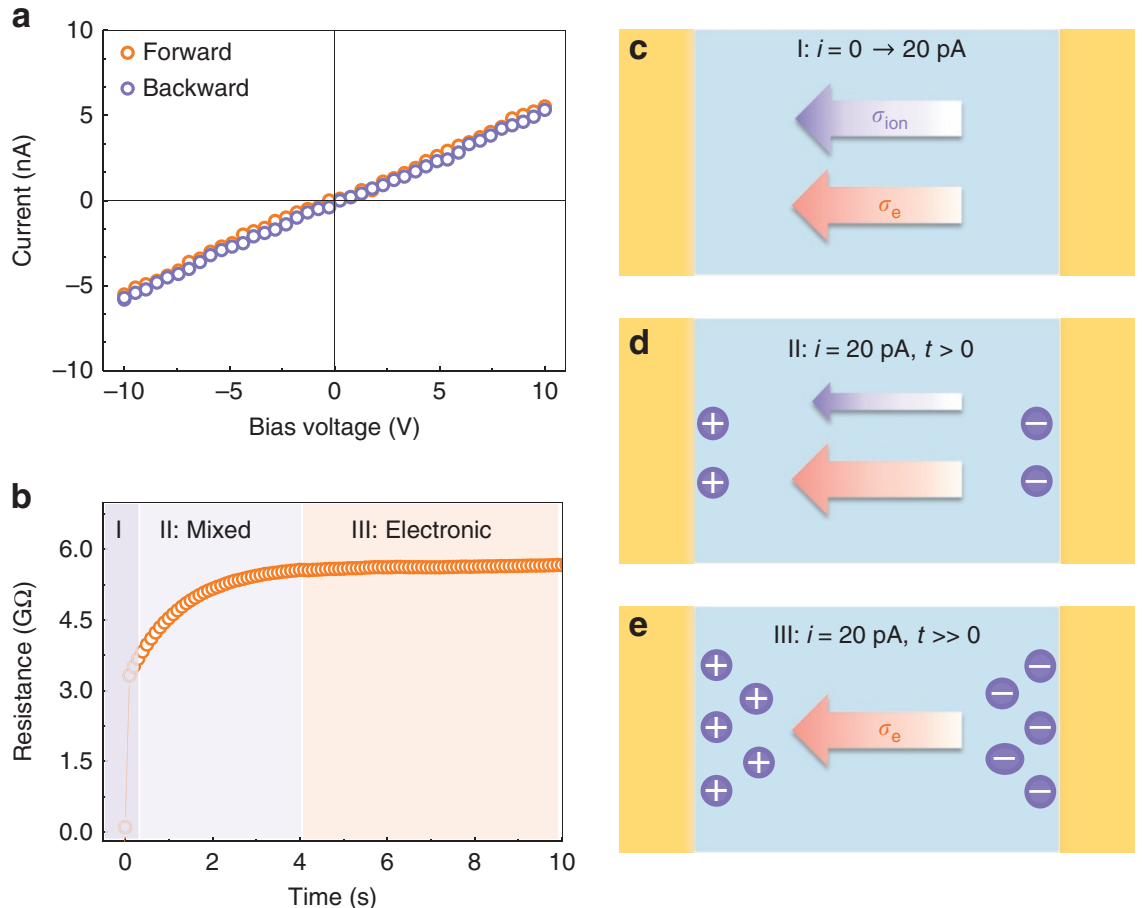

Figure 2 Characterization method for separating the ionic conductance from the mixed conductance in the perovskite film. (a) Voltage-current (V-I) measurements of the Au/perovskite/Au lateral device at $290 \mathrm{~K}$ under dark conditions at a $50 \mathrm{~V} \mathrm{~s}^{-1}$ scan rate. (b) Galvanostatic characterization of the lateral structure under dark conditions. A 20 pA current was switched on at $0.1 \mathrm{~s}$. Region II corresponds to the gradual depletion of the mobile ions, and region III corresponds to the pure electronic conductance in the perovskite film. (c) Schematic illustration of the conductance contributions from both ions and electrons, without any ionic depletion in the perovskite film (Region I). (d) Schematic illustration of the gradual depletion of mobile ions as they accumulate at the two sides of film, leading to a reduced ionic contribution to the conductance of the perovskite film (Region II). (e) Schematic illustration showing only electrons contributing to the conductance after the mobile ions in the film have been totally depleted at a sufficiently weak current of $20 \mathrm{pA}$, corresponding to the application of a $\sim 0.1-\mathrm{V}$ bias to the $50-\mu \mathrm{m}$ perovskite film (Region III).

constant, $I_{0}$ is the light intensity, and $f\left(I_{0}\right)$ is the carrier concentration generated via photoexcitation at an excitation power of $I_{0}$. Without photoexcitation $\left(f\left(I_{0}\right)=0\right)$, the conductivity flip is very weak because of the extremely low carrier concentration near $100 \mathrm{~K}$. However, under constant illumination, the second term dominates the conductivity near $100 \mathrm{~K}$, where $\mu(\mathrm{T})$ manifests itself by photogenerated carriers. Using the above formula, the experimental electronic conductivity data can be well fitted from 150 to $20 \mathrm{~K}$ (solid lines in Figure 3c).

The temperature-dependent ionic conductivity is plotted in Figure 3d. Unlike the exponential law that governs the electronic conductivity with respect to $1000 / \mathrm{T}, \ln \left(\sigma_{\text {ion }}\right)$ shows a much less clear behavior in the activation region, especially under photoexcitation. Unlike the band-like transport observed for electrons, the formula that describes the hopping-like ionic transport depends on the energy barrier $E_{\mathrm{a}}{ }^{27}$ :

$$
\sigma_{\text {ion }}=n e \mu=\frac{Z_{\mathrm{i}} e^{2} N_{\mathrm{A}} C_{\mathrm{v} 0} D_{0}}{k_{\mathrm{B}} T V_{\mathrm{m}}} \exp \left(-\frac{G_{\mathrm{v}}}{5 k_{\mathrm{B}} T}\right) \exp \left(-\frac{E_{\mathrm{a}}}{k_{\mathrm{B}} T}\right)
$$

Through transformation, we obtain

$$
\sigma_{\text {ion }} T=n e \mu=\frac{Z_{\mathrm{i}} e^{2} N_{\mathrm{A}} C_{\mathrm{v} 0} D_{0}}{k_{\mathrm{B}} V_{\mathrm{m}}} \exp \left(-\frac{G_{\mathrm{v}}}{5 k_{\mathrm{B}} T}-\frac{E_{\mathrm{a}}}{k_{\mathrm{B}} T}\right)=\sigma_{0} \exp \left(\frac{E_{\mathrm{a}}^{\text {eff }}}{K_{\mathrm{B}} T}\right)
$$

where $Z_{\mathrm{i}}$ is the ionic charge, $N_{\mathrm{A}}$ is Avogadro's constant, $C_{\mathrm{v}}$ is the concentration of intrinsic defects, $k_{\mathrm{B}}$ is the Boltzmann constant, $V_{\mathrm{m}}$ is the molar volume of perovskite, $D$ is the diffusion coefficient, $G_{\mathrm{v}}$ is the formation energy for vacancy defects, $E_{\mathrm{a}}$ is the activation energy for ionic diffusion, and $E_{\mathrm{a}}^{\mathrm{eff}}$ is defined to consider an excess vacancy formation energy in a vacancy-mediated mechanism. This formula shows many differences from the band-like electronic transport behavior and suggests that $\ln \left(\sigma_{\text {ion }} T\right)$, rather than $\ln \left(\sigma_{\text {ion }}\right)$, should be plotted versus 1000/T (Figure 3e). Linear regions from 140 to $295 \mathrm{~K}$ are observed in this plot, and the corresponding slopes markedly decrease with stronger illumination, reflecting the reduction in the activation energy for ionic transport. A zoomed-in view of the activation region (Figure 3f) further reveals two separate linear regions of the ionic conductance, $E_{\mathrm{a} 1}(\mathrm{~T}>250 \mathrm{~K})$ and $E_{\mathrm{a} 2}(180<\mathrm{T}<250 \mathrm{~K})$, which are summarized in Table 1 and offer quantitative evidence for light-dependent ion migration.

The reported activation energies for $\mathrm{I}^{-}$and $\mathrm{MA}^{+}$migration range from 0.1 to $1 \mathrm{eV}^{8-10,24}$. $\mathrm{H}^{+}$migration is theoretically predicted to have an activation energy of $0.17 \mathrm{eV}$, which will decrease as a result of nuclear quantum tunneling ${ }^{7}$. The $E_{\mathrm{a} 1}$ values obtained in our measurements range from 0.82 to $0.14 \mathrm{eV}$ and can thus be assigned to $\mathrm{I}^{-}$or $\mathrm{MA}^{+}$, whereas the $E_{\mathrm{a} 2}$ values are quite small, from 0.13 to $0.06 \mathrm{eV}$, and therefore should be assigned to $\mathrm{H}^{+}$. Further experimental evidence of proton migration via quantum tunneling is indicated by the crossover point of the kinetic constant near $100 \mathrm{~K}$, where the effects of quantum tunneling and thermal hopping meet ${ }^{26}$. This behavior will be discussed in our future work. The generation of protons should be closely related to $\mathrm{MA}^{+}$. An $\mathrm{H}^{+}$ion is attracted by the lone pair of nitrogen atom in $\mathrm{CH}_{3} \mathrm{NH}_{2}$, where $\mathrm{H}^{+}$acts as a Lewis acid, leading to a charge transfer interaction; however, the resulting bond can be broken by 
a

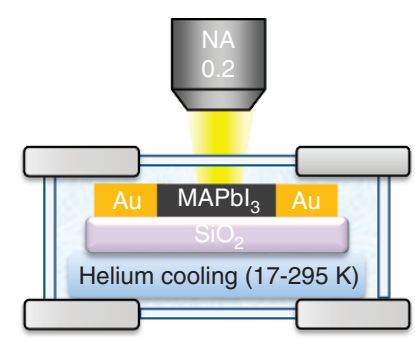

d

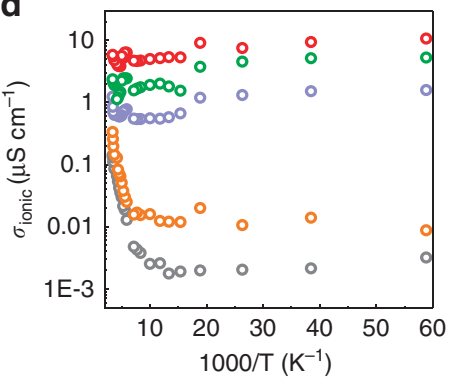

b

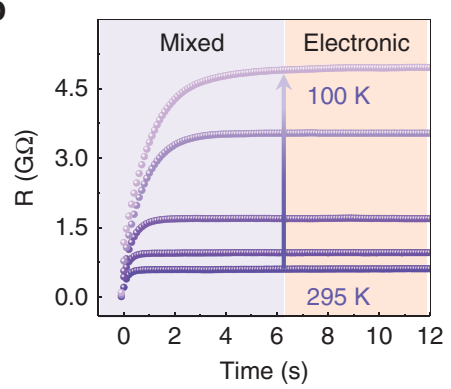

e

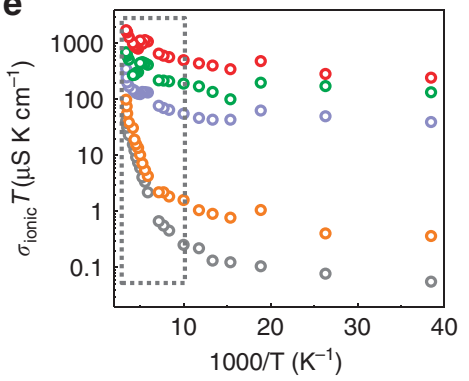

c

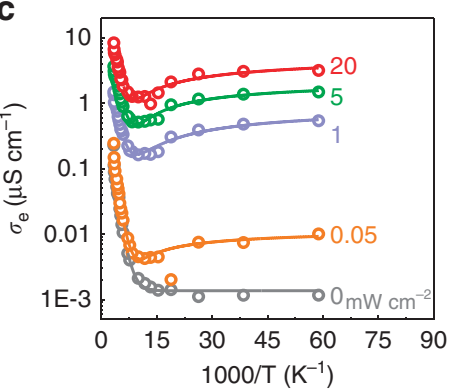

f

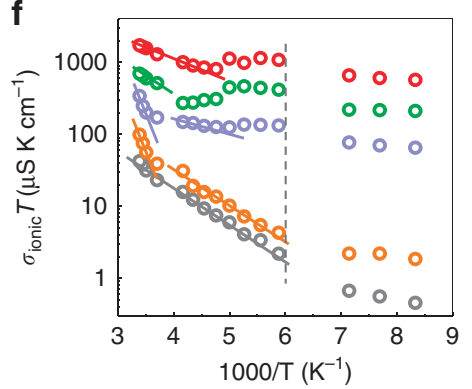

Figure 3 Determination of electronic and ionic conductivities of perovskite film at various temperatures and various illumination intensities from 0 to $20 \mathrm{~mW} \mathrm{~cm}^{-2}$. (a) The apparatus used in our experiments, with a helium cooling system. Our lateral device was prepared on a silica substrate with Au electrodes and mounted on a sample stage with a cooling system. (b) Five typical galvanostatic curves (resistance vs time) recorded at different temperatures under a $20 \mathrm{pA}$ current and $1 \mathrm{~mW} \mathrm{~cm}{ }^{-2}$ illumination. The temperatures from bottom to top are 295, 240, 200, 150 and $100 \mathrm{~K}$, respectively. (c) Electronic conductivities at various temperatures from 17 to $295 \mathrm{~K}$ as a function of 1000/T under various illumination intensities. The solid lines represent the fitted results for the electronic conductivity from 150 to $17 \mathrm{~K}$. (d) Ionic conductivities at various temperatures from 17 to $295 \mathrm{~K}$ under various illumination intensities. (e) Ionic conductivity multiplied by temperature, $\sigma_{\text {ion }} T$, as a function of 1000/T under various illumination intensities. (f) Zoomed-in view of the data in the dashed box in e. The dashed line indicates the phase transition temperature. Note that the ionic conductivity under $5 \mathrm{~mW} \mathrm{~cm}^{-2}$ illumination has been multiplied by 2 to distinguish the green dots from the purple dots in the plot.

Table 1 Summary of the activation energies extracted from the ionic/ electronic conductivities under different light intensities for $\mathrm{MAPbl}_{3}$

\begin{tabular}{lccc}
\hline $\begin{array}{l}\text { Light intensity } \\
\left.(\mathrm{mW} \mathrm{cm})^{-2}\right)\end{array}$ & $\begin{array}{c}E_{\mathrm{a} 1}(T>250 \mathrm{~K}) \\
(\mathrm{meV})\end{array}$ & \begin{tabular}{c}
$E_{\mathrm{a} 2}\left(\begin{array}{c}180<T<250 \mathrm{~K}) \\
(\mathrm{meV})\end{array}\right.$ \\
\hline 0 \\
Null
\end{tabular} & $\begin{array}{c}E_{\mathrm{a}} \text { (electronic) } \\
(\mathrm{meV})\end{array}$ \\
0.05 & 824 & 84 & 92 \\
1 & 851 & 63 & 84 \\
5 & 334 & Null & 73 \\
20 & 144 & Null & 44 \\
\hline
\end{tabular}

Note: The linear behavior from 180 to $250 \mathrm{~K}$ breaks down when the excitation power exceeds $5 \mathrm{~mW} \mathrm{~cm}{ }^{-2}$ at the phase transition point, thus making it impossible to extract the

corresponding activation energies at 5 and $20 \mathrm{~mW} \mathrm{~cm}-2$ for the ionic conductance in the temperature range from 180 to $250 \mathrm{~K}$.

thermal perturbation. For both $\mathrm{I}^{-} / \mathrm{MA}^{+}$and $\mathrm{H}^{+}$, the activation energy shows a marked reduction as the illumination intensity increases (Table 1), consistent with the increased ionic conductivity in perovskites under stronger illumination. To the best of our knowledge, this result is the first demonstration of light-dependent ionic transport in organometal halide perovskites.

Unlike the electronic conductance ${ }^{10,29}$, we observe that the ionic motion is influenced by phase transition (Supplementary Fig. S4), as indicated by a jump in the conductivity at $\sim 190 \mathrm{~K}$ (Figure $3 \mathrm{f}$ ). This phenomenon should be ascribed to the light-induced enhancement of the ion migration behavior caused by the reduced activation energy: the change in the energy barrier due to the phase transition results in a larger degree of influence on ionic transport with a smaller activation energy. In addition, the disappearance of the linear region corresponding to $E_{\mathrm{a} 1}(\mathrm{~T}>250 \mathrm{~K})$ under dark condition may be related to a larger activation energy under dark condition, which should be observed at higher temperatures.

A structural transformation of the perovskite, a change in the valence of the ions or a weakened bond strength of the $\mathrm{MA}^{+}$ may explain the reduction in the migration barrier under illumination ${ }^{2,7,20-22}$. Theoretical calculations show that differently charged defects allow ionization-enhanced migration and that photoexcitation may modulate the defects' charge, thereby influencing ionic transport ${ }^{7}$. Recent work has also revealed that polar molecules can greatly influence $\mathrm{MA}^{+}$motion in perovskites because of the reduced bond strength between $\mathrm{MA}^{+}$and adjacent $\mathrm{I}^{-}$cations ${ }^{2}$. Hence, illumination may also weaken the hydrogen bonding of $\mathrm{MA}^{+}$by means of photo-induced carriers, thereby influencing the ionic motion in perovskites ${ }^{21,30}$.

\section{Light-induced phenomena in metal halide perovskites}

Light-dependent ionic transport has important implications for the effects of light exposure on perovskite solar cells, such as the photoinduced halide segregation ${ }^{20,31}$, the giant dielectric constant ${ }^{23}$, and the photo-instability of the long-term output ${ }^{16}$. We present a schematic diagram of the enhanced ion migration under illumination in Figure 4a. Under the same external field, the ionic accumulation is accelerated by light exposure because of the increase in the diffusion coefficient, $D=D_{0} \exp \left(-\frac{E_{\mathrm{a}}}{K_{\mathrm{B}} T}\right)$. Therefore, ionic transport becomes easier when the light intensity increases. The observed halide segregation behavior under illumination in mixed $\mathrm{MAPb}\left(\mathrm{I}_{\mathrm{x}} \mathrm{Br}_{1-\mathrm{x}}\right)_{3}$ can be understood as an effect of light-enhanced ionic transport. For $\mathrm{MAPb}$ 
$\left(\mathrm{I}_{0.5} \mathrm{Br}_{0.5}\right)_{3}$, the initial PL peak at $640 \mathrm{~nm}$ splits into two peaks $(\sim 650$ and $750 \mathrm{~nm}$ ) under illumination (Figure $4 \mathrm{~b}$ ), accompanied by a monotonic increase in the PL intensity near $750 \mathrm{~nm}$ over time. These finding indicate that ion migration and redistribution occur when the sample is illuminated. However, this process is not fully reversible if the samples are stored under dark conditions for $60 \mathrm{~min}$; only a slight blue shift is observed in the PL (Figure 4c). Phenomenologically, we speculate that light can induce a bonding transformation, thereby changing the Gibbs energy-concentration relation (Figure 4d), reducing the miscibility under light exposure. Meanwhile, because of the reduced energy barrier, ions move rapidly toward more stable sites to minimize the total Gibbs energy, causing the $\mathrm{MAPb}\left(\mathrm{I}_{0.5} \mathrm{Br}_{0.5}\right)_{3}$ to separate into $\mathrm{MAPb}\left(\mathrm{I}_{\mathrm{x}} \mathrm{Br}_{1-\mathrm{x}}\right)_{3}$ with various doping concentrations. Although the miscibility of the mixed perovskites returns to a high level after storage under dark conditions, $E_{\mathrm{a}}$ also immediately increases, thereby suppressing ionic motion; consequently, the perovskite tends to remain in a metastable state. The light-induced giant dielectric constant of this material is also related: since chemical capacitance can be induced by ionic motion in the low-frequency regime, a larger dielectric constant will result from the stronger ionic motion under illumination, $C \propto \frac{\varepsilon_{\mathrm{r}}}{d}$.

Finally, we discuss the photo-instability observed in the stabilized power conversion efficiency (PCE) of perovskite solar cells in a glove box, which is among the greatest challenges hindering the commercialization of perovskite solar cells. It is well known that perovskite solar cells demonstrate worse stability under illumination than under dark conditions, even in the absence of decomposition induced by moisture or oxygen ${ }^{16}$. Moreover, this fundamental degradation mechanism is strongly related to the illumination intensity, as shown in Figure 4e. The steady-state PCE exhibits only a slight decrease under illumination at 0.5 or $4.5 \mathrm{~mW} \mathrm{~cm}^{-2}$, but it drops to $\sim 70 \%$ of its initial value during the first $100 \mathrm{~s}$ when the light intensity is increased to 30 or $100 \mathrm{~mW} \mathrm{~cm}^{-2}$. We speculate that under strong illumination, more ions tend to migrate toward the two sides of the device because of the reduced energy barrier and that the charge transfer is consequently suppressed by these excess defects ${ }^{2,8,10,13}$. Therefore, additional concerns regarding the stability of perovskite solar cells under illumination should be raised by the light-dependent ionic transport in $\mathrm{MAPbI}_{3}$.

\section{Light-assisted sequential deposition}

Considering that ionic interdiffusion is involved in the reaction between $\mathrm{PbI}_{2}$ and MAI stacking layers fabricated via sequential deposition, the limited ionic transport in the vertical direction usually results in excess $\mathrm{PbI}_{2}$ residue ${ }^{32}$. Microstructured $\mathrm{PbI}_{2}$ has been proposed to solve this problem in previous studies ${ }^{33-35}$. Herein, inspired by the observation of light-enhanced ion migration, we developed a novel light-assisted sequential deposition method to reduce excess $\mathrm{PbI}_{2}$. The procedures are illustrated in Figure 5a. After MAI is spin coated onto a prepared uniform film of $\mathrm{PbI}_{2}$, the subsequent annealing process is assisted by $40 \mathrm{~mW} \mathrm{~cm}^{-2}$ light exposure. Three batches of samples were produced for comparison, corresponding to 0,5 and $10 \mathrm{~min}$ of light exposure during the annealing process $\left(110^{\circ} \mathrm{C} / 10 \mathrm{~min}\right)$. The XRD patterns show evidence of a significant reduction in $\mathrm{PbI}_{2}$ residue $\left(2 \theta=12.6^{\circ}\right)$ in the case of light-assisted sequential deposition (Figure 5b). As a result, the
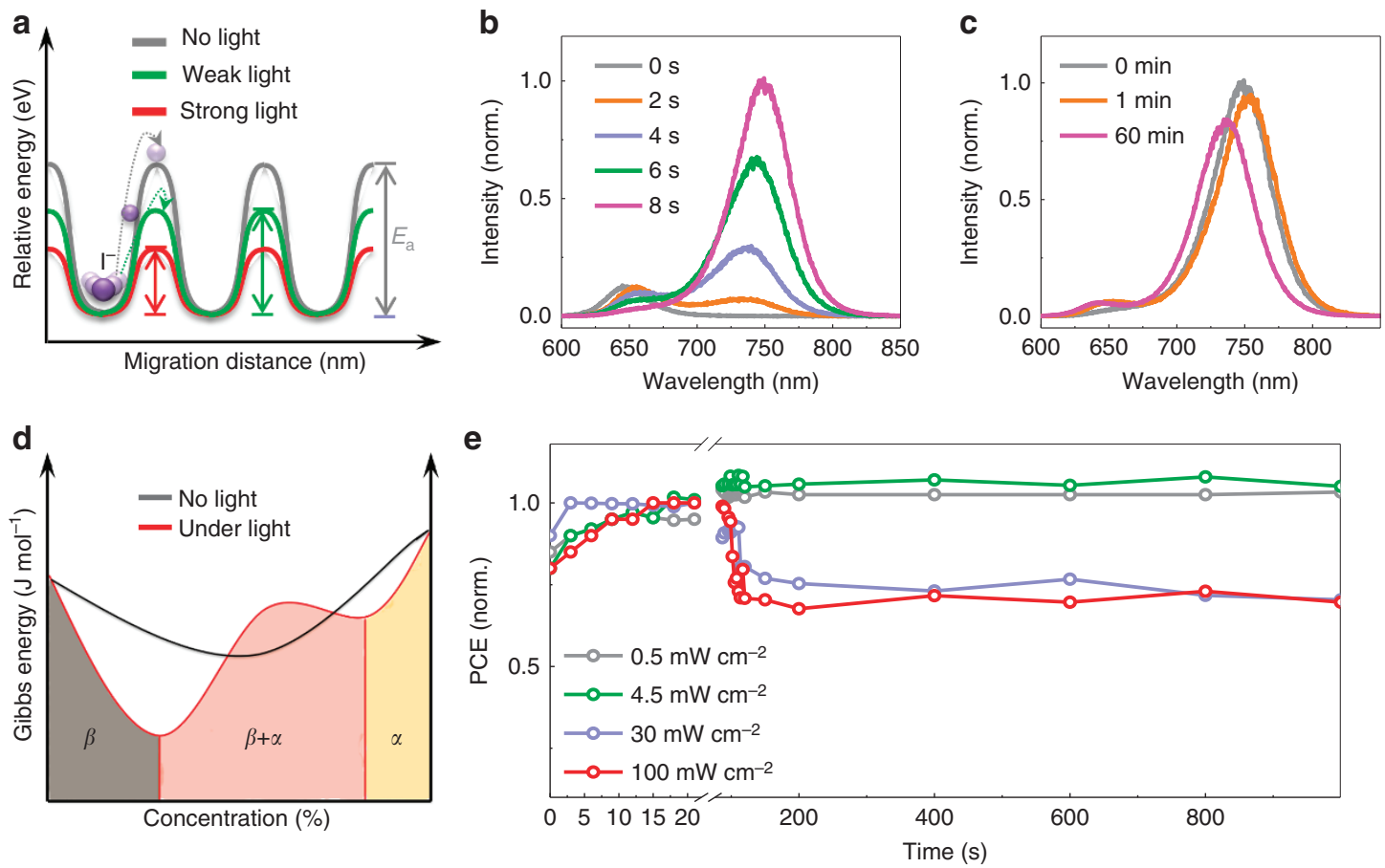

Figure 4 Photo-induced effects in perovskite films and devices. (a) Schematic illustration of light-dependent ion migration in perovskite MAPbl 3 . (b) Photoluminescence spectra of the mixed perovskite $\mathrm{MAPb}\left(\mathrm{I}_{0.5} \mathrm{Br}_{0.5}\right)_{3}$ after various illumination durations. (c) Photoluminescence spectra of the mixed perovskite $\mathrm{MAPb}\left(\mathrm{I}_{0.5} \mathrm{Br}_{0.5}\right)_{3}$ after subsequent storage under dark conditions for 1 and 60 min. (d) Schematic plot of the Gibbs energy as a function of the doping concentration for $\mathrm{MAPb}\left(\mathrm{I}_{\mathrm{x}} \mathrm{Br}_{1-\mathrm{x}}\right)_{3}$ under dark and light conditions. $\beta(14 / \mathrm{mcm})$ represents $\mathrm{Br}$ doping in the tetragonal $\mathrm{MAPb}_{3}$ matrix, and $\alpha(P m \overline{3} m)$ represents I doping in the cubic $\mathrm{MAPbBr}_{3}$ matrix. (e) Light-soaking stability tests under various illumination conditions. The devices were tested in an uncontaminated glove box at room temperature and were held at the maximum power point at $0.7 \mathrm{~V}$, and the data were directly obtained from the steadystate values. 
a
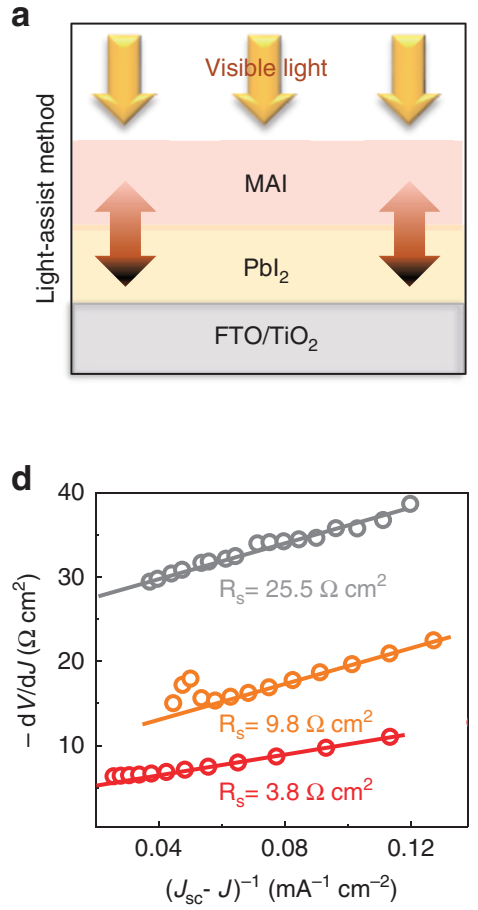

b

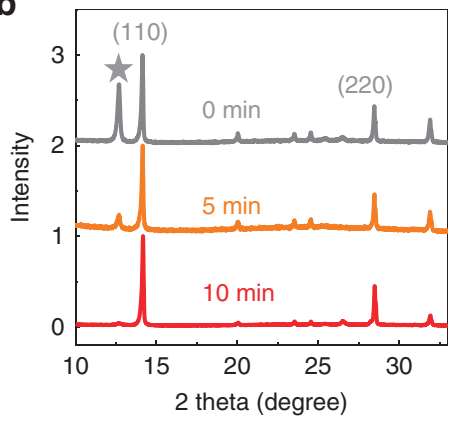

e

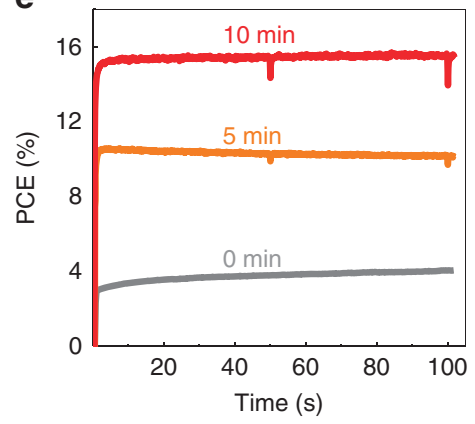

C

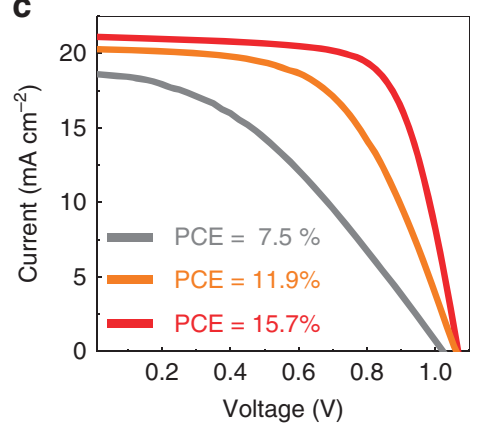

f

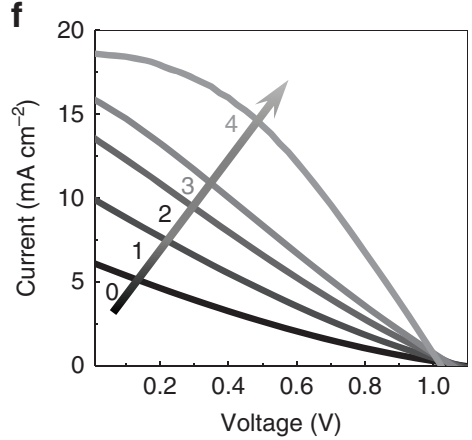

Figure 5 Light-assisted sequential deposition. (a) Schematic illustration of light-assisted sequential deposition: during the annealing process, visible light is shed on the sample through an 800-nm short-pass filter. (b) XRD patterns of the as-prepared perovskite films without light exposure (0 min), with 5 min of light exposure $(5 \mathrm{~min})$ and with $10 \mathrm{~min}$ of light exposure $(10 \mathrm{~min})$ during an otherwise identical 10 -min annealing process. (c) Current-voltage (I-V) measurements of solar cells fabricated using the corresponding films prepared with or without light exposure during the annealing process. (d) Plots of $d V / d J$ vs $\left(J_{S C}-J^{-1}\right.$ and the linear fit curves for these cells. (e) Steady-state performance of these devices at the maximum power point $(0.55,0.75$ and $0.83 \mathrm{~V}$ represents for 0,5 and $10 \mathrm{~min}$ of light exposure, respectively). (f) For the cell fabricated without light-assisted sequential deposition, the changes in the I-V curves during a continuous measurement under AM1.5 illumination. The values $0,1,2,3$ and 4 represent the light exposure durations, during which the cell was under open-circuit conditions.

corresponding device efficiency is increased from 7.5 to $15.7 \%$ with the reduction in $\mathrm{PbI}_{2}$ (Figure $5 \mathrm{c}$ ). The series resistances $R_{\mathrm{S}}$ of the cells were derived from the intercepts of the linear fitting results for plots of $-\mathrm{d} V / \mathrm{d} J$ vs $\left(J_{\mathrm{SC}}-J\right)^{-1}$, where $J$ and $J_{\mathrm{sc}}$ are the current density and short-circuit current density, respectively ${ }^{36}$. The derived series resistance was found to decrease from 25.5 to $3.8 \Omega \mathrm{cm}^{2}$ (Figure $5 \mathrm{~d}$ ) as a result of light-assisted synthesis, indicating that the reduction in interfacial $\mathrm{PbI}_{2}$ enhances the charge transfer process. The steadystate performance at the maximum power point reveals an extremely slow response of the control device $(0 \mathrm{~min})$ when the light is switched on (Figure 5e). This slow process is consistent with the gradual improvement in the $\mathrm{I}-\mathrm{V}$ measurements with increasing illumination duration observed for the control device (Figure 5f), in which stability is reached only after $4 \mathrm{~min}$ of light exposure. To detect the location of the residual $\mathrm{PbI}_{2}$, we used time-of-flight secondary-ionmass spectrometry (ToF-SIMS) to obtain the chemical depth profiles for lead and iodide (Supplementary Fig. S5), which revealed that these substances are mainly located at the bottom of the film. Therefore, excess $\mathrm{PbI}_{2}$ at the bottom of the film will hinder charge transfer, and we speculate that, facilitated or activated by light, $\mathrm{MA}^{+} / \mathrm{I}^{-}$ will slowly move and react with $\mathrm{PbI}_{2}$ under a chemical potential gradient. This slow reaction leads to the gradual improvement in charge transfer behavior observed here. Therefore, the results presented above further support the observation of light-enhanced ionic transport.

\section{CONCLUSIONS}

Light-dependent ionic transport has been quantitatively demonstrated in perovskite film over a wide range of illumination intensities by separating the ionic conductivity from the mixed conductivity. Light exerts a significant influence on the ionic transport in $\mathrm{MAPbI}_{3}$ by reducing the activation energies for $\mathrm{I}^{-} / \mathrm{MA}^{+}$and $\mathrm{H}^{+}$. This property of $\mathrm{MAPbI}_{3}$ has important implications for the photo-induced halide segregation, giant dielectric constant, and unstable behavior observed in perovskite films and devices. A light-assisted synthesis method is proposed to control the formation of $\mathrm{PbI}_{2}$ residues in perovskites to ensure better film quality by means of the light-enhanced ionic interdiffusion process. Further attention should be paid to the entire perovskite family, such as in $\mathrm{NH}_{2} \mathrm{CH}=\mathrm{NHPbI}_{3}$ and $\mathrm{CsPb}_{3}$, especially for inorganic system, to determine the role of organic component in light-induced effects. Our findings also imply a complicated interplay between light conditions and optoelectronic properties in excited state perovskite materials as a result of ion redistribution, thus making it urgent to investigate the microscopic mechanisms of these behaviors.

\section{CONFLICT OF INTEREST}

The authors declare no conflict of interest.

\section{ACKNOWLEDGEMENTS}

This work was supported by the National 973 Project (2013CB932602, MOST) of the Ministry of Science and Technology of China and the National Natural Science Foundation of China (NSFC51272007, 61571015, 11327902, 11234001, 
91433102 and 51522201). QZ acknowledges the Beijing Nova Program (XX2013003) and the Program for New Century Excellent Talents in University of China.

1 Xiao ZG, Yuan YB, Shao YC, Wang Q, Dong QF et al. Giant switchable photovoltaic effect in organometal trihalide perovskite devices. Nat Mater 2015; 14: 193-198.

2 Leijtens T, Hoke ET, Grancini G, Slotcavage DJ, Eperon GE et al. Mapping electric fieldinduced switchable poling and structural degradation in hybrid lead halide perovskite thin films. Adv Energy Mater 2015; 5: 1500962.

3 Azpiroz JM, Mosconi E, Bisquert J, de Angelis F. Defect migration in methylammonium lead iodide and its role in perovskite solar cell operation. Energy Environ Sci 2015; 8 : 2118-2127.

4 Leijtens T, Eperon GE, Noel NK, Habisreutinger SN, Petrozza A et al. Stability of metal halide perovskite solar cells. Adv Energy Mater 2015; 5: 1500963.

5 Leijtens T, Srimath Kandada AR, Eperon GE, Grancini G, D'Innocenzo V et al. Modulating the electron-hole interaction in a hybrid lead halide perovskite with an electric field. J Am Chem Soc 2015; 137: 15451-15459.

6 Yang TY, Gregori G, Pellet N, Grätzel M, Maier J. The significance of ion conduction in a hybrid organic-inorganic lead-iodide-based perovskite photosensitizer. Angew Chem Int Ed Engl 2015; 54: 7905-7910.

7 Egger DA, Kronik L, Rappe AM. Theory of hydrogen migration in organic-inorganic halide perovskites. Angew Chem Int Ed Eng/ 2015; 54: 12437-12441.

8 Eames C, Frost JM, Barnes PRF, O'Regan BC, Walsh A et al. Ionic transport in hybrid lead iodide perovskite solar cells. Nat Commun 2015; 6: 7497.

9 Meloni S, Moehl T, Tress W, Franckevičius M, Saliba M et al. Ionic polarization-induced current-voltage hysteresis in $\mathrm{CH}_{3} \mathrm{NH}_{3} \mathrm{PbX}_{3}$ perovskite solar cells. Nat Commun 2016; 7: 10334.

10 Brenner TM, Egger DA, Kronik L, Hodes G, Cahen D. Hybrid organic-inorganic perovskites: low-cost semiconductors with intriguing charge-transport properties. Nat Rev Mater 2016; 1: 15007.

$11 \mathrm{Xu}$ JX, Buin A, Ip AH, Li W, Voznyy $\mathrm{O}$ et al. Perovskite-fullerene hybrid materials suppress hysteresis in planar diodes. Nat Commun 2015; 6: 7081.

12 Zhao YC, Zhou WK, Ma W, Meng S, Li H et al. Correlations between immobilizing ions and suppressing hysteresis in perovskite solar cells. ACS Energy Lett 2016; 1: 266-272.

$13 \mathrm{Cho} \mathrm{H}$, Jeong SH, Park MH, Kim YH, Wolf $\mathrm{C}$ et al. Overcoming the electroluminescence efficiency limitations of perovskite light-emitting diodes. Science 2015; 350: 1222-1225.

14 Zhu HM, Fu YP, Meng F, Wu XX, Gong ZZ et al. Lead halide perovskite nanowire lasers with low lasing thresholds and high quality factors. Nat Mater 2015; 14: 636-642.

15 Wei HT, Fang YJ, Mulligan P, Chuirazzi W, Fang HH et al. Sensitive X-ray detectors made of methylammonium lead tribromide perovskite single crystals. Nat Photonics 2016; 10: 333-339.

16 Zhao YC, Wei J, Li H, Yan Y, Zhou WK et al. A polymer scaffold for self-healing perovskite solar cells. Nat Commun 2016; 7: 10228.

17 Kim HS, Mora-Sero I, Gonzalez-Pedro V, Fabregat-Santiago F, Juarez-Perez EJ et al. Mechanism of carrier accumulation in perovskite thin-absorber solar cells. Nat Commun 2013; 4: 2242.

18 Deng YH, Xiao ZG, Huang JS. Light-induced self-poling effect on organometal trihalide perovskite solar cells for increased device efficiency and stability. Adv Energy Mater 2015; 5: 1500721.

19 Lee JW, Kim DH, Kim HS, Seo SW, Cho SM et al. Formamidinium and cesium hybridization for photo- and moisture-stable perovskite solar cell. Adv Energy Mater 2015; 5: 1501310.

20 Hoke ET, Slotcavage DJ, Dohner ER, Bowring AR, Karunadasa HI et al. Reversible photo-induced trap formation in mixed-halide hybrid perovskites for photovoltaics. Chem Sci 2015; 6: 613-617.
21 Gottesman R, Gouda L, Kalanoor BS, Haltzi E, Tirosh S et al. Photoinduced reversible structural transformations in free-standing $\mathrm{CH}_{3} \mathrm{NH}_{3} \mathrm{Pbl}_{3}$ perovskite films. J Phys Chem Lett 2015; 6: 2332-2338.

22 Gottesman R, Haltzi E, Gouda L, Tirosh S, Bouhadana $Y$ et al. Extremely slow photoconductivity response of $\mathrm{CH}_{3} \mathrm{NH}_{3} \mathrm{Pbl}_{3}$ perovskites suggesting structural changes under working conditions. J Phys Chem Lett 2014; 5: 2662-2669.

23 Juarez-Perez EJ, Sanchez RS, Badia L, Garcia-Belmonte G, Kang YS et al. Photoinduced giant dielectric constant in lead halide perovskite solar cells. J Phys Chem Lett 2014; 5: 2390-2394.

24 Yokota I. On the theory of mixed conduction with special reference to the conduction in silver sulfide group semiconductors. J Physical Soc Japan 1961; 16: 2213-2223.

25 Yuan YB, Chae J, Shao YC, Wang Q, Xiao ZG et al. Photovoltaic switching mechanism in lateral structure hybrid perovskite solar cells. Adv Energy Mater 2015; 5: 1500615.

26 Yuan YB, Wang Q, Shao YC, Lu HD, Li T et al. Electric-field-driven reversible conversion between methylammonium lead triiodide perovskites and lead iodide at elevated temperatures. Adv Energy Mater 2016; 6: 1501803.

27 Mizusaki J, Arai K, Fueki K. Ionic conduction of the perovskite-type halides. Solid State Ion 1983; 11: 203-211.

28 Yokota I. On the electrical conductivity of cuprous sulfide: a diffusion theory. J Physical Soc Japan 1953; 8: 595-602

29 Oga H, Saeki A, Ogomi Y, Hayase S, Seki S. Improved understanding of the electronic and energetic landscapes of perovskite solar cells: high local charge carrier mobility, reduced recombination, and extremely shallow traps. J Am Chem Soc 2014; 136: $13818-13825$.

30 Wu XJ, Yu H, Li LK, Wang F, Xu HH et al. Composition-dependent light-induced dipole moment change in organometal halide perovskites. J Phys Chem C 2015; 119: 1253-1259.

31 McMeekin DP, Sadoughi G, Rehman W, Eperon GE, Saliba M et al. A mixed-cation lead mixed-halide perovskite absorber for tandem solar cells. Science 2016; 351: $151-155$.

32 Xiao ZG, Bi C, Shao YC, Dong QF, Wang Q et al. Efficient, high yield perovskite photovoltaic devices grown by interdiffusion of solution-processed precursor stacking layers. Energy Environ Sci 2014; 7: 2619-2623.

33 Zhang $\mathrm{H}$, Mao J, He HX, Zhang D, Zhu HL et al. A smooth $\mathrm{CH}_{3} \mathrm{NH}_{3} \mathrm{Pbl}_{3}$ film via a new approach for forming the $\mathrm{Pbl}_{2}$ nanostructure together with strategically high $\mathrm{CH}_{3} \mathrm{NH}_{3} \mathrm{I}$ concentration for high efficient planar-heterojunction solar cells. Adv Energy Mater 2015; 5: 1501354

$34 \mathrm{Liu} \mathrm{TH}, \mathrm{Hu} \mathrm{Q}$, Wu J, Chen K, Zhao LC et al. Mesoporous $\mathrm{Pbl}_{2}$ scaffold for highperformance planar heterojunction perovskite solar cells. Adv Energy Mater 2016; 6: 1501890.

35 Wu CG, Chiang CH, Tseng ZL, Nazeeruddin MK, Hagfeldt A et al. High efficiency stable inverted perovskite solar cells without current hysteresis. Energy Environ Sci 2015; 8: 2725-2733.

36 Shi JJ, Dong J, Lv ST, Xu YZ, Zhu LF et al. Hole-conductor-free perovskite organic lead iodide heterojunction thin-film solar cells: high efficiency and junction property. Appl Phys Lett 2014; 104: 063901.

(c) (i) $\odot$ This work is licensed under a Creative Commons Attributioncy NC ND NonCommercial-NoDerivs 4.0 International License. The images or other third party material in this article are included in the article's Creative Commons license, unless indicated otherwise in the credit line; if the material is not included under the Creative Commons license, users will need to obtain permission from the license holder to reproduce the material. To view a copy of this license, visit http:// creativecommons.org/licenses/by-nc-nd/4.0/

(C) The Author(s) 2017

Supplementary Information for this article can be found on the Light: Science \& Applications' website (http://www.nature.com/lsa). 